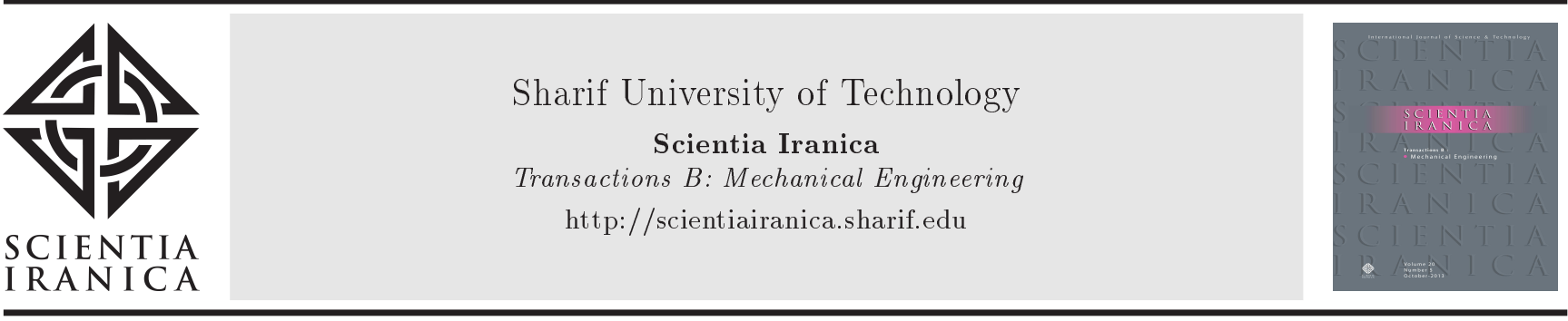

\title{
Analytical solution for coupled non-Fickian diffusion-thermoelasticity and thermoelastic wave propagation analysis
}

\author{
S.A. Hosseini ${ }^{\mathrm{a}}$, S.M. Hosseini ${ }^{\mathrm{b}}$, and M.H. Abolbashari $\mathrm{i}^{\mathrm{c}, *}$ \\ a. Department of Mechanical Engineering, Faculty of Engineering, Ferdowsi University of Mashhad, Mashhad, P.O. Box \\ 917r75-1111, Iran. \\ b. Department of Industrial Engineering, Faculty of Engineering, Ferdowsi University of Mashhad, Mashhad, P.O. Box 917775-1111, \\ Iran. \\ c. Lean Production Engineering Research Center, Department of Mechanical Engineering, Ferdowsi University of Mashhad, \\ Mashhad, P.O. Box 91775-1111, Iran.
}

Received 7 January 2016; received in revised form 24 June 2017; accepted 13 August 2017

\author{
KEYWORDS \\ Non-Fickian diffusion; \\ Wave propagation; \\ Molar concentration; \\ Temperature; \\ Analytical method; \\ Coupled problems.
}

\begin{abstract}
The time history analysis and propagation of molar concentration, temperature, and displacement waves are studied in details using an analytical method. The method is applied to coupled non-Fickian diffusion-thermoelasticity analysis of a strip. The governing equations are derived using non-Fickian theory of diffusion and classic theories for coupled thermoelasticity. Molar concentration and thermoelastic wave propagations are considered to be of finite speed. The governing equations are first transferred to the frequency domain using Laplace transform technique. The unknown parameters are then obtained in analytical forms proposed by the presented method. By employing the Talbot technique, the unknown parameters are eventually determined in time domain. It can be concluded that the presented analytical method has a high capability for dynamic and transient analysis of coupled diffusion-thermoelasticity problems. The wave fronts in displacement, temperature, and molar concentration fields can be tracked at various time instants employing the presented analytical method.
\end{abstract}

(C) 2018 Sharif University of Technology. All rights reserved.

\section{Introduction}

Diffusion can be defined as the random walk of an ensemble of particles, from regions of high concentration to regions of lower concentration. The coupling between the thermoelastic fields and concentration of diffusive gas takes place when a solid body is immersed in a gas. Thermo-diffusion in an elastic solid is due

\footnotetext{
*. Corresponding author.

E-mail addresses: Amin.hoss@gmail.com (S.A. Hosseini); sm_hosseini@um.ac.ir (S.M.Hosseini); abolbash@um.ac.ir (M.H. Abolbashari).
}

doi: $10.24200 /$ sci.2017.4505 to the field of temperature, mass diffusion, and that of strain [1].

At present, much attention is paid to the governing equations and boundary conditions by using the chemical potential definition and the first and the second laws of thermodynamics. Recently, Kumar et al. [2] presented analytical solutions to the time fractional diffusion equation using Homotopy Perturbation Transform Method (HPTM). This method is a combined form of the Laplace transform and homotopy perturbation methods. The variational principle is usually used to derive a fully coupled multifield formulation [3-6]. The variational method has been regarded as the base of analysis and computations in the coupled fields. In non-Fickian theory of diffusion, it 
is assumed that the molar concentration is propagated with finite speed. There are some published works in which the non-Fickian diffusion problems have been studied using numerical methods [7-9]. Kuang $[3,10]$ proposed an inertial entropy concept and an inertial concentration or chemical potential concept. Also, Kuang [4] considered that variation of temperature should be supported by the extra heat and proposed an inertial entropy theory from which a temperature wave equation with a finite propagation velocity was obtained. So many generalized thermoelastic theories have been proposed to allow a finite velocity for the propagation of a thermal wave. There are mainly 4 generalized theories: Lord-Shulman (LS) [11], GreenLindsay (GL) [12], Green-Naghdi (GN) [13], and the inertial entropy [4].

In the recent years, some research works have been carried out for coupled thermoelasticity analysis based on the non-classical theories in coupled thermoelasticity using analytical $[14,15]$ as well as numerical $[16,17]$ methods.

Lee [18] presented the thermoelasticity problem of a multilayered hollow cylinder whose boundaries were subjected to time-dependent temperatures, adiabatic and clamped. In the case of an infinitely long cylinder, numerical results of multilayered hollow cylinder were calculated with finite difference and Laplace transform methods. In another research, Xia et al. [19] studied an isotropic semi-infinite plate subjected to a moving heat source by employing the finite element method directly in the time domain.

Sharyiat [20] carried out nonlinear generalized (with second sound effect) and classical thermoelasticity analyses for functionally graded thick cylinders subjected to various thermomechanical shocks at their inner and outer surfaces employing Hermitian elements. Recently, Sharyiat et al. [21,22] investigated some aspects of the vibration and wave propagation phenomena in thick-hollow functionally graded cylinders under thermomechanical loads using a numerical method. Bagri and Eslami [23,24] presented a unified formulation in which a few theories of coupled thermoelasticity were considered. They employed their unified formulation for dynamic and transient analysis of coupled thermoelasticity in cylinders. Sherief and Abd El-Latief [25] studied the fractional order theory of thermoelasticity for a 2D problem for a half-space and solved it with Laplace and exponential Fourier transforms techniques. Hosseini et al. [26] presented an analytical method for one-dimensional analysis of coupled non-Fickian diffusion and mechanics. Kumar and Chawla [27] studied the fundamental solution to two-dimensional problem in orthotropic thermodiffusive elastic medium. Some various problems for the theory of generalized thermoelastic diffusion have also been studied during the last few years [28-30]. The effects of rotation and diffusion on the plane-wave propagation were presented by Singh et al. [31] in which a thermoelastic half-space without energy dissipation problem was solved using GN theory (Type II). Allam et al. [32] studied a thermoelastic diffusion interaction in an infinitely long annular cylinder using the theory of thermoelastic diffusion with one relaxation time.

Recently, several variational principles for coupled temperature-diffusion-mechanics were presented by Sue and Shen [33]. In another work [34], they presented an approximate analytical method for onedimensional analysis of coupled non-Fickian diffusion and mechanics without considering temperature effects. Hosseini et al. [35] presented a 2D dynamic analysis of coupled non-Fickian diffusion-elasticity for a 2D domain in functionally graded materials using Meshless Local Petrov-Galerkin (MLPG) method. The propagation of wave fronts in both molar concentration and displacement fields were obtained at various times. In another work, Hosseini et al. [36] employed the MLPG method for 2D coupled non-Fickian diffusionthermoelasticity based on GN theory of coupled thermoelasticity. They studied the dynamic behaviors of molar concentrations, temperature, and displacement fields.

The main purpose of this work is to present an effective analytical method to study the coupled non-Fickian diffusion-thermoelasticity based on the inertial entropy theory [4] in a strip subjected to shock loading. The molar concentration, temperature, and displacements are obtained in analytical forms using the presented analytical method. In this way, the wave fronts of molar concentration, displacement, and temperature can be tracked at various time instants. It is assumed that the temperature, diffusion, and elastic waves are propagated at finite speeds.

\section{Governing equations}

The constitutive equations for the coupled non-Fickian diffusion-thermoelasticity can be written based on the classical theory of coupled thermoelasticity [33] in the following forms:

$$
\begin{aligned}
& \sigma_{i j}=C_{i j k l} \varepsilon_{k l}-\alpha_{i j} c-\beta_{1 i j}\left(T-T_{0}\right), \\
& \tilde{\mu}=\alpha_{i j} \varepsilon_{i j}+\beta c+\tau_{1} T, \\
& \tilde{s}=\beta_{1 i j} \varepsilon_{i j}+\tau_{1} c+\frac{A}{T_{0}} T,
\end{aligned}
$$

where $\sigma_{i j}, \varepsilon_{i j}, c, \tilde{s}, A, T$, and $\tilde{\mu}$ are the stress, strain, molar concentration, entropy, temperature constant, temperature, and chemical potential $(\mathrm{J} / \mathrm{mol})$, respectively. The terms $C_{i j k l}, \alpha_{i j}, \beta_{1 i j}, \tau_{1}$, and $\beta$ stand for the elastic coefficients, mechanical diffusion coefficient, 
mechanical-thermo coefficient, thermo-chemical coefficient, and the chemical potential constant, respectively.

The equation of motion in elasticity, the nonFickian general diffusion equations, and entropy balance equation can be taken into account as follows [33]:

$$
\begin{aligned}
& \sigma_{i j, j}+f_{i}=\rho \ddot{u}_{i}, \\
& \dot{\tilde{\mu}}+\dot{\tilde{\mu}}^{(a)}=\frac{I}{c_{0}}-\frac{\beta^{\prime} J_{i, i}}{c_{0}}, \\
& \dot{\tilde{s}}+\dot{\tilde{s}}^{(a)}=\frac{\dot{r}}{T}-\frac{q_{i, i}}{T},
\end{aligned}
$$

where $f_{i}, \rho, u_{i}, \tilde{\mu}^{(a)}, \tilde{s}^{(a)}, I, r, q_{i}$, and $c_{0}$ are the body force, density, displacement, inertial chemical potential, inertial entropy, diffusion source term, body heat source strength, the heat flow, and reference concentration, respectively. The term $J_{i}$ is the diffusion flux $\left(\mathrm{mol} / \mathrm{m}^{3} \mathrm{~s}\right)$, which satisfies $\mathbf{J}=-D_{0} \nabla \mathbf{c}$, and $D_{0}$ is the diffusion coefficient. $\tilde{\mu}^{(a)}$ and $\tilde{s}^{(a)}$ are assumed to be proportional to the rates of concentration and temperature, respectively. $\tilde{\mu}^{(a)}=\gamma \dot{c}$, and $\gamma$ is a coefficient. $\beta^{\prime}$ is equal to $R T$, where $R$ is the universal gas constant and $T$ is the absolute temperature. $\beta^{\prime}$ and $\beta$ satisfy $\beta=\beta^{\prime} / c_{0} . \tilde{s}^{(a)}=\frac{\hat{\hat{\omega}} \hat{\omega}}{T_{0}} \dot{T}, \hat{c}$ is the specific heat and $\hat{\omega}$ is a constant coefficient with dimension of time. Eqs. (2) can be rewritten in the following forms by neglecting the body force, diffusion source term, and body heat source strength:

$$
\begin{aligned}
& \sigma_{i j, j}=\rho \ddot{u}_{i}, \\
& \dot{\tilde{\mu}}+\dot{\tilde{\mu}}^{(a)}=-\frac{\beta^{\prime} J_{i, i}}{c_{0}}=-\beta J_{i, i}, \\
& \dot{\tilde{s}}+\dot{\tilde{s}}^{(a)}=-\frac{q_{i, i}}{T_{0}} .
\end{aligned}
$$

For a strip made of homogenous isotropic materials, the governing equations can be developed as follows:

$$
\begin{aligned}
& (2 \mu+\lambda) \frac{\partial^{2} u}{\partial x^{2}}-\alpha \frac{\partial c}{\partial x}-\beta_{1} \frac{\partial \theta}{\partial x}=\rho \frac{\partial^{2} u}{\partial t^{2}} \\
& \sigma=(2 \mu+\lambda) \frac{\partial u}{\partial x}-\alpha c-\beta_{1} \theta \\
& D_{0} \frac{\partial^{2} c}{\partial x^{2}}=\frac{\alpha}{\beta} \frac{\partial^{2} u}{\partial x \partial t}+\frac{\partial c}{\partial t}+\tau_{0} \frac{\partial^{2} c}{\partial t^{2}}+\frac{\tau_{1}}{\beta} \frac{\partial \theta}{\partial t} \\
& \frac{k}{T_{0}} \frac{\partial^{2} \theta}{\partial x^{2}}=\beta_{1} \frac{\partial^{2} u}{\partial x \partial t}+\tau_{1} \frac{\partial c}{\partial t}+\frac{\hat{c} \hat{\omega}}{T_{0}} \frac{\partial^{2} \theta}{\partial t^{2}}+\frac{A}{T_{0}} \frac{\partial \theta}{\partial t}
\end{aligned}
$$

where $\theta=T-T_{0}, \tau_{0}=\gamma / \beta$ is the relaxation time, and $\lambda$ and $\mu$ are Lame's constants. Eq. (4c) for molar concentration balance is concluded by combining Eq. (1) and Eq. (3) with the expressions of $J_{i}$ and $\tilde{\mu}^{(a)}$.
Obviously, there exist the coupled terms between the non-Fickian diffusion and thermoelasticity in Eqs. (4). It is noted that if the coupled term in Eq. (4c) is not considered, i.e., $\alpha=0$ and $\tau_{1}=0$, Eq. (4c) will become the pure non-Fickian diffusion.

The following homogenous initial conditions are assumed for the problem:

$$
\begin{aligned}
& u(x, t)=\frac{\partial u(x, t)}{\partial t}=0, \quad \text { at } t=0, \\
& c(x, t)=\frac{\partial c(x, t)}{\partial t}=0, \quad \text { at } t=0, \\
& \theta(x, t)=\frac{\partial \theta(x, t)}{\partial t}=0, \quad \text { at } t=0 .
\end{aligned}
$$

The governing equations are solved for a strip of which the boundary is excited by a shock loading for molar concentration and temperature in the form of Heaviside unit step function of time:

$$
\begin{array}{ll}
\sigma(x, t)=0, & \text { at } \quad x=0, \\
c(x, t)=c_{1} H(t), & \text { at } \quad x=0, \\
\theta(x, t)=\theta_{1} H(t), & \text { at } \quad x=0, \\
u(x, t)=0, & \text { when } \quad x=1.5 \mathrm{~m}, \\
c(x, t)=0, & \text { when } \quad x=1.5 \mathrm{~m}, \\
\theta(x, t)=0, & \text { when } \quad x=1.5 \mathrm{~m},
\end{array}
$$

where $H(t)$ is the Heaviside function.

The application of Laplace transform in Eqs. (4a), (4c), and (4d) with respect to time yields:

$$
\begin{aligned}
(2 \mu+\lambda) \frac{\partial^{2} \bar{u}(x, s)}{\partial x^{2}}-\alpha \frac{\partial \bar{c}(x, s)}{\partial x}-\beta_{1} \frac{\partial \bar{\theta}(x, s)}{\partial x} & \rho s^{2} \bar{u}(x, s), \\
D_{0} \frac{\partial^{2} \bar{c}(x, s)}{\partial x^{2}}= & \frac{\alpha}{\beta} s \frac{\partial \bar{u}(x, s)}{\partial x}+s \bar{c}(x, s) \\
& +\tau_{0} s^{2} \bar{c}(x, s)+\frac{\tau_{1}}{\beta} s \bar{\theta}(x, s), \\
\frac{k}{T_{0}} \frac{\partial^{2} \bar{\theta}(x, s)}{\partial x^{2}}= & \beta_{1} s \frac{\partial \bar{u}(x, s)}{\partial x}+\tau_{1} s \bar{c}(x, s) \\
& +\frac{\hat{c} \hat{\omega}}{T_{0}} s^{2} \bar{\theta}(x, s)+\frac{A}{T_{0}} s \bar{\theta}(x, s),
\end{aligned}
$$

where the terms $\bar{u}(x, s), \bar{c}(x, s)$, and $\bar{\theta}(x, s)$ are displacement, molar concentration, and temperature in Laplace domain. 
Also, the boundary conditions may be written in Laplace domain as:

$$
\begin{array}{ll}
\bar{\sigma}(x, s)=0, & \text { at } \quad x=0, \\
\bar{c}(x, s)=\frac{c_{1}}{s}, & \text { at } \quad x=0, \\
\bar{\theta}(x, s)=\frac{\theta_{1}}{s}, & \text { at } x=0, \\
\bar{u}(x, s)=0, & \text { when } x=1.5 \mathrm{~m}, \\
\bar{c}(x, s)=0, & \text { when } x=1.5 \mathrm{~m}, \\
\bar{\theta}(x, s)=0, & \text { when } x=1.5 \mathrm{~m} .
\end{array}
$$

To find the dynamic behaviors of molar concentration, temperature, and displacement from Eqs. (7)-(9), an analytical method is employed, which is explained in the next section.

\section{Analytical solution}

The solution to Eqs. (7), (8), and (9) can be expressed as the following Taylor series in terms of $(x-1)$; they are analytical at $(x-1)$ :

$$
\begin{aligned}
& \bar{u}(x, s)=\sum_{n=0}^{\infty} A_{n}(s)(x-1)^{n}, \\
& \bar{c}(x, s)=\sum_{n=0}^{\infty} B_{n}(s)(x-1)^{n}, \\
& \bar{\theta}(x, s)=\sum_{n=0}^{\infty} D_{n}(s)(x-1)^{n}
\end{aligned}
$$

where $A_{n}(s), B_{n}(s)$, and $D_{n}(s)$ are unknown coefficients. By substituting Eqs. (11), (12), and (13) in Eqs. (7), (8), and (9), the following equations can be derived:

$$
\begin{aligned}
(\lambda+2 \mu) & \sum_{n=2}^{\infty} A_{n}(s) n(n-1)(x-1)^{n-2} \\
& -\alpha \sum_{n=1}^{\infty} B_{n}(s) n(x-1)^{n-1} \\
& -\beta_{1} \sum_{n=1}^{\infty} D_{n}(s) n(x-1)^{n-1} \\
& -\rho s^{2} \sum_{n=0}^{\infty} A_{n}(s)(x-1)^{n}=0,
\end{aligned}
$$

$$
\begin{aligned}
& \frac{\alpha}{\beta} s \sum_{n=1}^{\infty} A_{n}(s) n(x-1)^{n-1}+s \sum_{n=0}^{\infty} B_{n}(s)(x-1)^{n} \\
& \quad+\tau_{0} s^{2} \sum_{n=0}^{\infty} B_{n}(s)(x-1)^{n}+\frac{\tau_{1}}{\beta} s \sum_{n=0}^{\infty} D_{n}(s)(x-1)^{n} \\
& \quad-D_{0} \sum_{n=2}^{\infty} B_{n}(s) n(n-1)(x-1)^{n-2}=0, \\
& \beta_{1} s \sum_{n=1}^{\infty} A_{n}(s) n(x-1)^{n-1}+\tau_{1} s \sum_{n=0}^{\infty} B_{n}(s)(x-1)^{n} \\
& \quad+\frac{A}{T_{0}} s \sum_{n=0}^{\infty} D_{n}(s)(x-1)^{n}+\frac{\hat{c} \hat{\omega}}{T_{0}} s^{2} \sum_{n=0}^{\infty} D_{n}(s)(x-1)^{n} \\
& \quad-\frac{k}{T_{0}} \sum_{n=2}^{\infty} D_{n}(s) n(n-1)(x-1)^{n-2}=0 .
\end{aligned}
$$

Eqs. (14)-(16) may be rewritten in the new forms of:

$$
\begin{aligned}
& \sum_{n=0}^{\infty}\left[(\lambda+2 \mu)(n+1)(n+2) A_{n+2}(s)\right. \\
& \quad-\alpha(n+1) B_{n+1}(s)-\beta_{1}(n+1) D_{n+1}(s) \\
& \left.\quad-\rho s^{2} A_{n}(s)\right](x-1)^{n}=0 \\
& \sum_{n=0}^{\infty}\left[\frac{\alpha}{\beta} s(n+1) A_{n+1}(s)+s B_{n}(s)+\tau_{0} s^{2} B_{n}(s)\right. \\
& \left.\quad+\frac{\tau_{1}}{\beta} s D_{n}(s)-D_{0}(n+1)(n+2) B_{n+2}(s)\right](x-1)^{n} \\
& \quad=0
\end{aligned}
$$$$
\sum_{n=0}^{\infty}\left[\beta_{1} s(n+1) A_{n+1}(s)+\tau_{1} s B_{n}(s)+\frac{A}{T_{0}} s D_{n}(s)\right.
$$$$
\left.+\frac{\hat{c} \hat{\omega}}{T_{0}} s^{2} D_{n}(s)-\frac{k}{T_{0}}(n+1)(n+2) D_{n+2}(s)\right](x-1)^{n}
$$$$
=0 .
$$

To find the unknown coefficients, the following recurrence relations can be derived as follows:

$$
\begin{aligned}
A_{n+2}(s)= & \frac{\alpha}{(n+2)(\lambda+2 \mu)} B_{n+1}(s) \\
& +\frac{\rho s^{2}}{(n+1)(n+2)(\lambda+2 \mu)} A_{n}(s) \\
& +\frac{\beta_{1}}{(n+2)(\lambda+2 \mu)} D_{n+1}(s),
\end{aligned}
$$




$$
\begin{aligned}
B_{n+2}(s)= & \frac{\alpha s}{\beta D_{0}(n+2)} A_{n+1}(s) \\
& +\frac{s+\tau_{0} s^{2}}{D_{0}(n+1)(n+2)} B_{n}(s) \\
& +\frac{\tau_{1} s}{\beta D_{0}(n+1)(n+2)} D_{n}(s), \\
D_{n+2}(s)= & \frac{T_{0} \beta_{1} s}{k(n+2)} A_{n+1}(s) \\
& +\frac{T_{0} \tau_{1} s}{k(n+1)(n+2)} B_{n}(s) \\
& +\frac{A s+\hat{c} \hat{\omega} s^{2}}{k(n+1)(n+2)} D_{n}(s) .
\end{aligned}
$$

It can be seen in Eqs. (21), (22), and (23) that all coefficients $A_{n}(s), B_{n}(s)$, and $D_{n}(s)$ can be expressed in terms of " $A_{0}(s)$ ", " $A_{1}(s)$ ", " $B_{0}(s)$ ", " $B_{1}(s)$ ", " $D_{0}(s)$ ", and " $D_{1}(s)$ " when " $n>1$ ". The terms " $A_{0}(s)$ ", " $A_{1}(s)$ ", " $B_{0}(s)$ ", " $B_{1}(s), " D_{0}(s)$ ", and " $D_{1}(s)$ " should be determined using boundary conditions. Therefore, the solutions (11), (12), and (13) can be rewritten in new forms as:

$$
\begin{aligned}
\bar{u}(x, s)= & \sum_{n=0}^{\infty}\left\{g_{1}^{n}(s) A_{0}(s)+g_{2}^{n}(s) A_{1}(s)\right. \\
& +g_{3}^{n}(s) B_{0}(s)+g_{4}^{n}(s) B_{1}(s)+g_{5}^{n}(s) D_{0}(s) \\
& \left.+g_{6}^{n}(s) D_{1}(s)\right\}(x-1)^{n} \\
\bar{c}(x, s)= & \sum_{n=0}^{\infty}\left\{K_{1}^{n}(s) A_{0}(s)+K_{2}^{n}(s) A_{1}(s)\right. \\
& +K_{3}^{n}(s) B_{0}(s)+K_{4}^{n}(s) B_{1}(s)+K_{5}^{n}(s) D_{0}(s) \\
& \left.+K_{6}^{n}(s) D_{1}(s)\right\}(x-1)^{n}, \\
\bar{\theta}(x, s)= & \sum_{n=0}^{\infty}\left\{L_{1}^{n}(s) A_{0}(s)+L_{2}^{n}(s) A_{1}(s)\right. \\
& +L_{3}^{n}(s) B_{0}(s)+L_{4}^{n}(s) B_{1}(s)+L_{5}^{n}(s) D_{0}(s) \\
& \left.+L_{6}^{n}(s) D_{1}(s)\right\}(x-1)^{n} .
\end{aligned}
$$

The coefficients " $g_{i}^{n}(s)$ ", " $K_{i}^{n}(s)$ ", and " $L_{i}^{n}(s)$ " when " $i=1$ to 6 " may be calculated using the following equations:

$$
\begin{aligned}
g_{i}^{n+2}(s)= & \frac{\alpha}{(n+2)(\lambda+2 \mu)} K_{i}^{n+1}(s) \\
& +\frac{\rho s^{2}}{(n+1)(n+2)(\lambda+2 \mu)} g_{i}^{n}(s)
\end{aligned}
$$

$$
\begin{aligned}
& +\frac{\beta_{1}}{(n+2)(\lambda+2 \mu)} L_{i}^{n+1}(s), \\
k_{i}^{n+2}(s)= & \frac{s+\tau_{0} s^{2}}{D_{0}(n+1)(n+2)} K_{i}^{n}(s) \\
& +\frac{\alpha s}{\beta D_{0}(n+2)} g_{i}^{n+1}(s) \\
& +\frac{\tau_{1} s}{\beta D_{0}(n+1)(n+2)} L_{i}^{n}(s), \\
L_{i}^{n+2}(s)= & \frac{A s+\hat{c} \hat{\omega} s^{2}}{k(n+1)(n+2)} L_{i}^{n}(s)+\frac{T_{0} \beta_{1} s}{k(n+2)} g_{i}^{n+1}(s) \\
& +\frac{T_{0} \tau_{1} s}{k(n+1)(n+2)} k_{i}^{n}(s) .
\end{aligned}
$$

Also:

$$
\begin{aligned}
& g_{1}^{0}(s)=1, \quad g_{2}^{0}(s)=0, \quad g_{3}^{0}(s)=0, \\
& g_{4}^{0}(s)=0, \quad g_{5}^{0}(s)=0, \quad g_{6}^{0}(s)=0, \\
& g_{1}^{1}(s)=0, \quad g_{2}^{1}(s)=1, \quad g_{3}^{1}(s)=0, \\
& g_{4}^{1}(s)=0, \quad g_{5}^{1}(s)=0, \quad g_{6}^{1}(s)=0, \\
& g_{1}^{2}(s)=\frac{\rho s^{2}}{2(\lambda+2 \mu)}, \quad g_{2}^{2}(s)=0, \quad g_{3}^{2}(s)=0 \\
& g_{4}^{2}(s)=\frac{\alpha}{2(\lambda+2 \mu)}, \quad g_{5}^{2}(s)=0 \\
& g_{6}^{2}(s)=\frac{\beta_{1}}{2(\lambda+2 \mu)}, \\
& K_{1}^{0}(s)=0, \quad K_{2}^{0}(s)=0, \quad K_{3}^{0}(s)=1, \\
& K_{4}^{0}(s)=0, \quad K_{5}^{0}(s)=0, \quad K_{6}^{0}(s)=0, \\
& K_{1}^{1}(s)=0, \quad K_{2}^{1}(s)=0, \quad K_{3}^{1}(s)=0, \\
& K_{4}^{1}(s)=1, \quad K_{5}^{1}(s)=0, \quad K_{6}^{1}(s)=0 \\
& K_{1}^{2}(s)=0, \quad K_{2}^{2}(s)=\frac{\alpha s}{2 \beta D_{0}} \\
& K_{3}^{2}(s)=\frac{s+\tau_{0} s^{2}}{2 D_{0}}, \quad K_{4}^{2}(s)=0 \\
& K_{5}^{2}(s)=\frac{\tau_{1} s}{2 \beta D_{0}}, K_{6}^{2}(s)=0
\end{aligned}
$$


as well as:

$$
\begin{aligned}
& L_{1}^{0}(s)=0, \quad L_{2}^{0}(s)=0, \quad L_{3}^{0}(s)=0, \\
& L_{4}^{0}(s)=0, \quad L_{5}^{0}(s)=1, \quad L_{6}^{0}(s)=0, \\
& L_{1}^{1}(s)=0, \quad L_{2}^{1}(s)=0, \quad L_{3}^{1}(s)=0, \\
& L_{4}^{1}(s)=0, \quad L_{5}^{1}(s)=0, \quad L_{6}^{1}(s)=1, \\
& L_{1}^{2}(s)=0, \quad L_{4}^{2}(s)=0, \\
& L_{3}^{2}(s)=\frac{\tau_{1} s T_{0} s}{2 k}, \quad L_{6}^{2}(s)=0 . \\
& L_{5}^{2}(s)=\frac{A s+\hat{c} \hat{\omega} s^{2}}{2 k}, \quad
\end{aligned}
$$

Eqs. (23)-(25) are the analytical solutions for displacement, molar concentration, and temperature fields in Laplace domain. To determine the solutions in time domain, the present work uses the Talbot algorithm [37], which is based on deforming the contour in the Bromwich inversion integral to reduce numerical error. This formulation yields relations in time domain as follows:

$$
\begin{aligned}
& u(x, t)=\frac{2}{5 t} \sum_{k=0}^{M-1} \operatorname{Re}\left(\gamma_{k} \bar{u}\left(x, s_{k}\right)\right), \\
& c(x, t)=\frac{2}{5 t} \sum_{k=0}^{M-1} \operatorname{Re}\left(\gamma_{k} \bar{c}\left(x, s_{k}\right)\right), \\
& \theta(x, t)=\frac{2}{5 t} \sum_{k=0}^{M-1} \operatorname{Re}\left(\gamma_{k} \bar{\theta}\left(x, s_{k}\right)\right),
\end{aligned}
$$

where:

$$
\begin{aligned}
s_{k} & =\frac{\delta_{k}}{t}, \quad \delta_{0}=\frac{2 M}{5}, \quad \gamma_{0}=0.5 e^{\delta_{0}} \\
\delta_{k} & =\frac{2 k \pi}{5}\left(\cot \left(\frac{k \pi}{M}\right)+i\right) \\
\gamma_{k} & =\left[1+i\left(\frac{k \pi}{M}\right)\left(1+\left[\cot \left(\frac{k \pi}{M}\right)\right]^{2}\right)-i \cot \left(\frac{k \pi}{M}\right)\right] e^{\delta_{k}} \\
0 & <k<M
\end{aligned}
$$

\section{Numerical results and discussion}

In this section, some numerical results are presented for the problem. For the sake of comparison, some of the material specifications of the problem are given as in [34]. However, material specifications and other
Table 1. Material specifications and other parameters of the problem.

$$
\begin{array}{ll}
\lambda=1.326 \times 10^{9}\left(\frac{\mathrm{N}}{\mathrm{m}^{2}}\right) & \mu=0.884 \times 10^{9}\left(\frac{\mathrm{N}}{\mathrm{m}^{2}}\right) \\
\rho=2000\left(\frac{\mathrm{kg}}{\mathrm{m}^{3}}\right) & D_{0}=10000\left(\frac{\mathrm{m}^{2}}{\mathrm{~s}}\right) \\
\beta=5832\left(\frac{\mathrm{N} \cdot \mathrm{m}^{4}}{\mathrm{~mol}^{2}}\right) & \alpha=1.87082\left(\frac{\mathrm{N} \cdot \mathrm{m}}{\mathrm{mol}}\right) \\
\tau_{0}=3.086 \times 10^{-1}(\mathrm{~s}) & \alpha_{t}=1.78 \times 10^{-5}\left(\frac{1}{{ }^{\circ} \mathrm{K}}\right) \\
k=1000\left(\frac{\mathrm{N}}{{ }^{\circ} \mathrm{K} . \mathrm{s}}\right) & A=1.8\left(\frac{\mathrm{N}}{\mathrm{m}^{2} \cdot{ }^{\circ} \mathrm{K}}\right) \\
\tau_{1}=0.0001\left(\frac{\mathrm{N} \cdot \mathrm{m}}{\mathrm{mol} .{ }^{\circ} \mathrm{K}}\right) & \hat{c} \hat{\omega}=24.5\left(\frac{\mathrm{N} \cdot \mathrm{s}}{\mathrm{m}^{2}{ }^{\circ} \mathrm{K}}\right) \\
c_{1}=93540\left(\frac{\mathrm{mol}}{\mathrm{m}^{3}}\right) & T_{0}=300\left({ }^{\circ} \mathrm{K}\right) \\
\beta_{1}=(3 \lambda+2 \mu) \alpha_{t} & \theta_{1}=450\left({ }^{\circ} \mathrm{K}\right)
\end{array}
$$

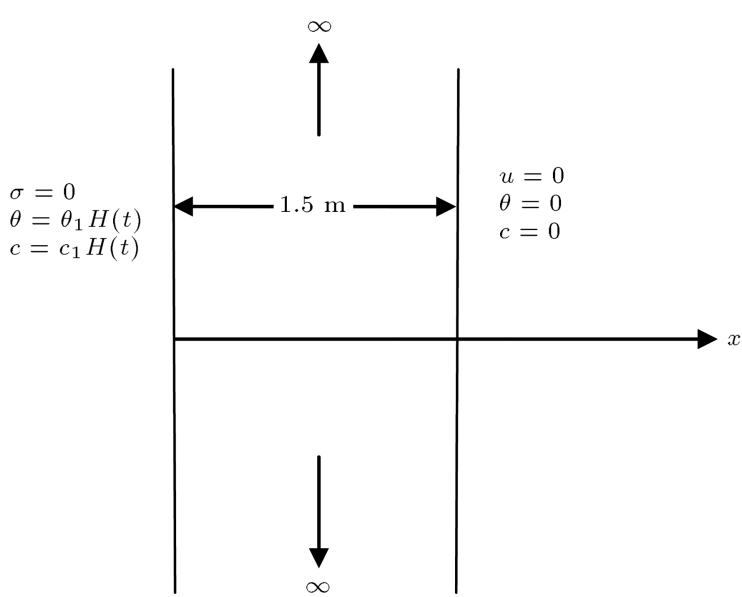

Figure 1. Schematic of the boundary conditions for the strip.

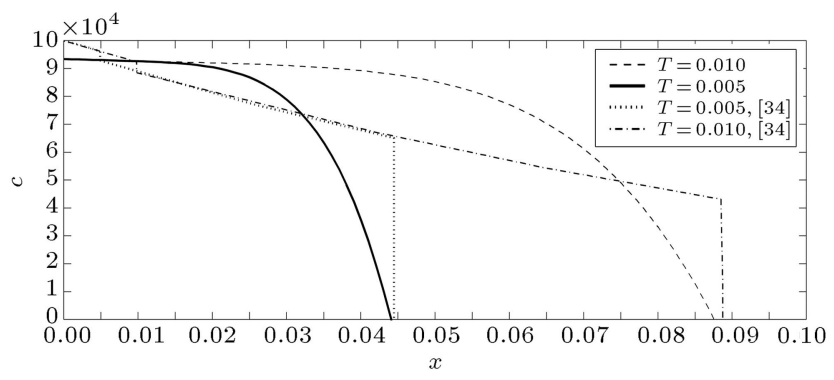

Figure 2. The comparison between obtained results and those of Ref. [34] for molar concentration along the $x$ direction.

parameters are presented in Table 1. The boundary conditions are illustrated in Figure 1.

For validation, a comparison between the molar concentration obtained by the proposed analytical method without considering temperature and those obtained in [33] is presented in Figure 2. The comparison reveals a good agreement. Therefore, the proposed analytical method can be considered as an effective analytical method with a high capability for solving coupled governing equations of this type and similar types. The variations of displacement, temperature, and molar concentration in time domain 


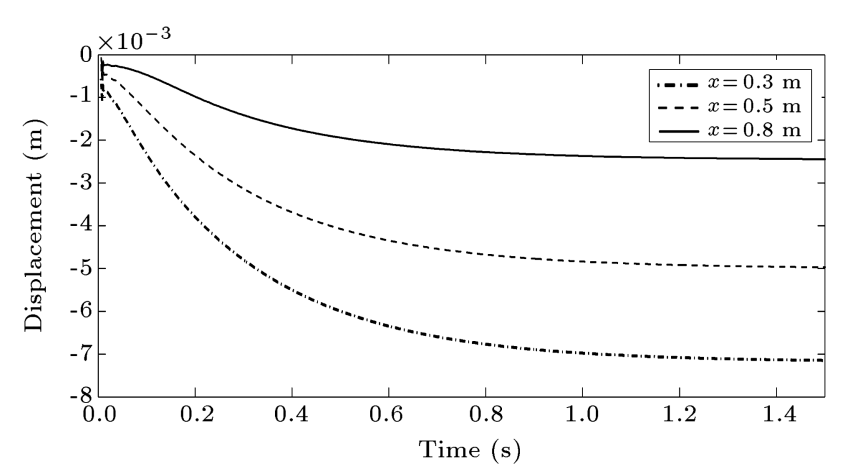

Figure 3. The dynamic behavior of the displacement at different positions in time domain.

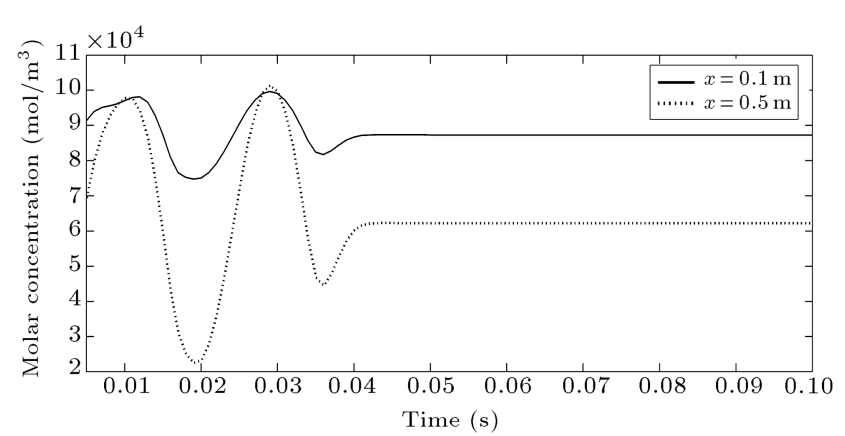

Figure 4. The dynamic behavior of the molar concentration at different positions in time domain.

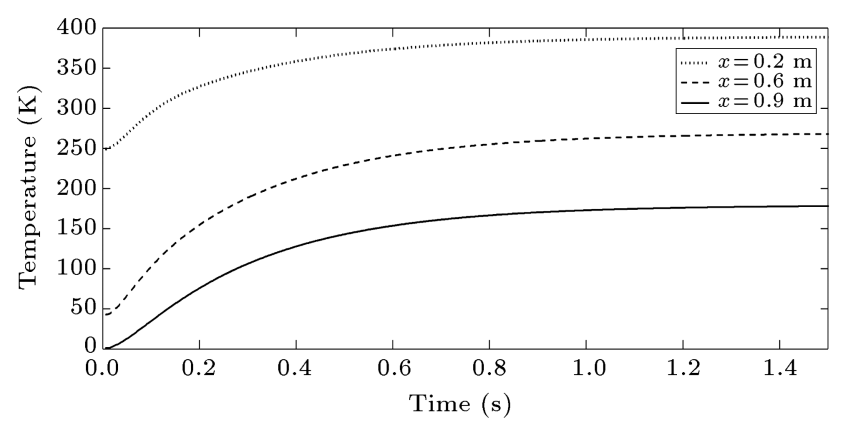

Figure 5. The dynamic behavior of the temperature at different positions in time domain.

are respectively presented in Figures 3, 4, and 5 for different positions. The transient as well as steady state behaviors are shown in these figures. The conversion between thermal and mechanical energy is the reason of disturbances in the transient parts of these diagrams. From time histories of displacement, temperature, and molar concentration, it is clearly concluded that the presented analytical method has an acceptable convergence rate. The distributions of molar concentration along the $x$ direction are shown in Figure 6. The propagation of wave front can be observed with finite speed of propagation. Also, as shown in this figure, with increasing the diffusion time, the diffusion distance gradually increases and, at the same time, concentration decreases. The distributions

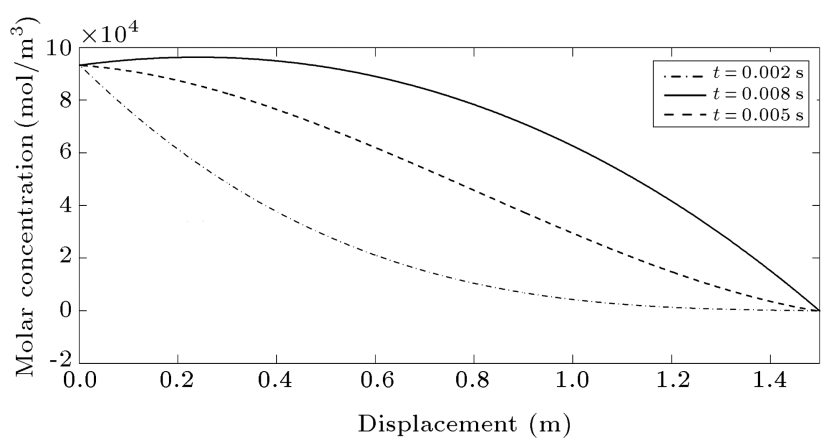

Figure 6. The wave fronts of molar concentration wave propagation along the $x$ direction for various time instants.

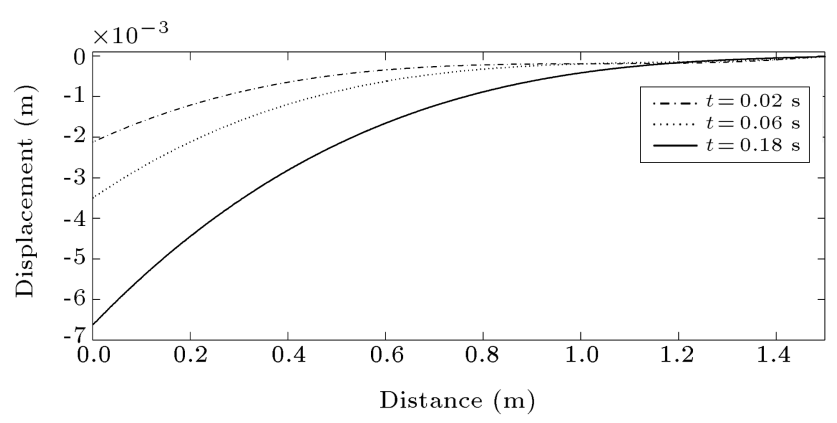

Figure 7. The wave fronts of displacement wave propagation along the $x$ direction for various time instants.

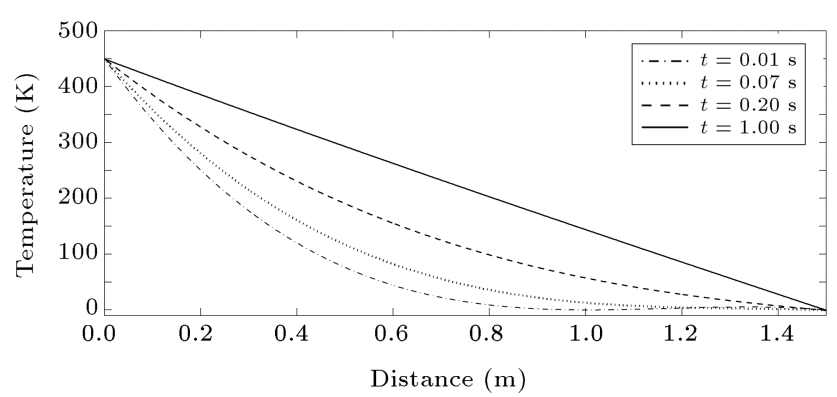

Figure 8. The wave fronts of temperature wave propagation along the $x$ direction for various time instants.

of displacement and temperature along the $x$ direction are illustrated in Figures 7 and 8 at various time instants. Obviously, as the diffusion distance increases, the displacement gradually approaches zero. It is also seen that the assumed mechanical boundary conditions are satisfied at each side of domain. It means that the presented method has a high convergence rate for satisfying the boundary conditions. As it can be observed in Figures 6-8, there are not any disturbances in the vicinity of wave fronts or other situations in diagrams. The reason may be the superiority of the convergence rate and high accuracy of the presented analytical method. The concentration field from the coupled non-Fick/non-classic diffusionthermoelasticity theory and that from the coupled classical diffusion-thermoelasticity are compared in Figure 9. It is obviously seen that at shorter times, 


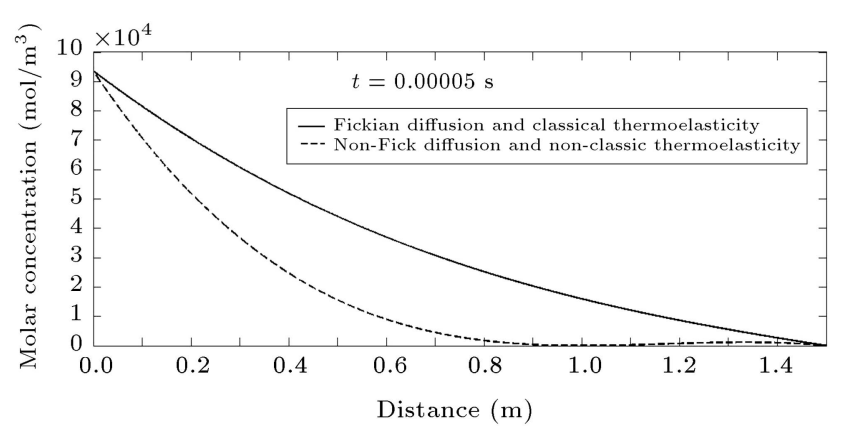

Figure 9. The comparison between classical and non-classical form of concentration along the $x$ direction.

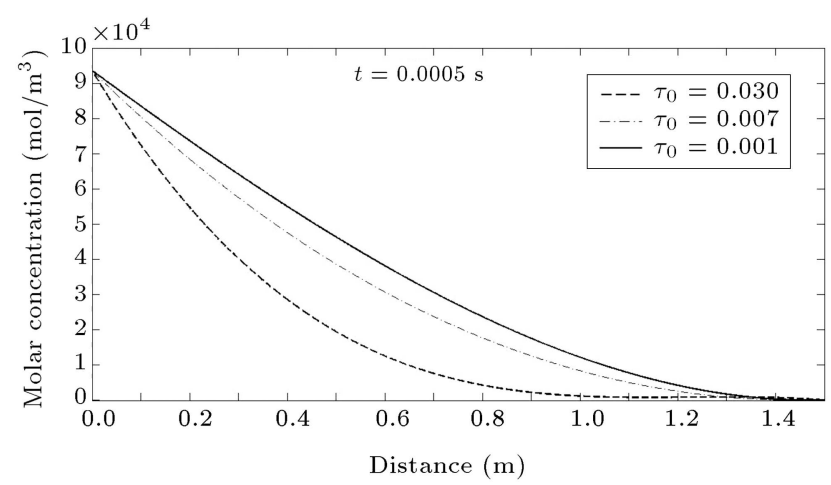

Figure 10. The effect of relaxation time on concentration along the $x$ direction.

the difference between the two solutions is significant. The effect of the diffusion relaxation time, $\tau_{0}$, on the velocity of wave propagation in molar concentration field can be observed in Figure 10. As it is shown, when the relaxation time decreases, the wave propagation velocities decrease.

\section{Conclusions}

In this article, an analytical solution was presented for the transient analysis of coupled non-Fickian diffusionthermo-elasticity based on the classical theory of coupled thermoelasticity. The coupled governing equations were transferred to Laplace domain and, then, the unknown parameters including temperature, molar concentration, and displacement were obtained in closed form in Laplace domain. Using the Talbot inversion Laplace technique, the dynamic behaviors were illustrated in time domain. The main conclusions of this study can be outlined as follows:

- The presented solution may be effectively used for studying the wave propagations of displacement, molar concentration, and temperature fields. The molar concentration and displacement wave fronts can be tracked using the presented analytical method at every arbitrary time instant;

- The time history and dynamic behaviors of displacement, molar concentration, and temperature are analyzed using an analytical method. Also, the effects of shock loadings of both molar concentration and temperature fields on the dynamic behaviors are obtained;

- The presented analytical solution and series forms for molar concentration and displacement furnish a suitable framework for optimization of coupled nonFickian diffusion-thermoelasticity problems.

\section{Nomenclature}

$A$

Temperature constant

$A_{n}(s)$,

$B_{n}(s)$,

$D_{n}(s)$

$c$

$\hat{c}$

$c_{0}$

$c_{1}$

$C_{i j k l}$

$D_{0}$

$f_{i}$

\section{$I$}

$J_{i}$

$k$

$q_{i}$

$r$

$R$

$\tilde{s}$

$\tilde{S}^{(a)}$

$\hat{\omega}$

$t$

T

$T_{0}$

$u_{i}$

$u_{i}$
$x$

$x$
$\alpha_{i j}, \alpha$

$\alpha_{i j}$,

$\beta_{1 i j}, \beta_{1}$

$\beta$

$\gamma$

$\lambda, \mu$

$\tilde{\mu}$

$\tilde{\mu}^{(a)}$

$\rho$

$\sigma_{i j}$

$\tau_{0}$

Unknown coefficients

Mass concentration

Specific heat

Reference concentration

Shock concentration

The elastic constants

Diffusion coefficient

Body force

Diffusion source

Diffusion flux

Thermal conductivity

Heat flow

Body heat source strength

Universal gas constant

Entropy

Proportional to the rate of temperature

Constant coefficient

Time

Absolute temperature

Reference temperature

Components of displacement vector

Position

Mechanical diffusion coefficient

Coefficient of linear thermal expansion

Mechanical-thermo coefficient

Chemical potential constant

Constant coefficient

Lame's constants

Chemical potential

Proportional to the rate of concentration

Density

$\sigma_{i j} \quad$ Components of stress tensor

Relaxation time 


\section{References}

1. Levine, I.N., Physical Chemistry, 6th Ed., McGraw Hill, New York (2009).

2. Kumar, S., Yildirim, A., Khan, Y., and Wei, L. "A fractional model of the diffusion equation and its analytical solution using Laplace transform", Scientia Iranica B, 19(4), pp. 1117-1123 (2012).

3. Kuang, Z.B. "Some variational principles in elastic dielectric and elastic magnetic material", European Journal of Mechanics-A/Solids, 27, pp. 504-514 (2008).

4. Kuang, Z.B. "Variational principles of generalized dynamical theory of thermopiezoelectricity", Acta Mech., 203, pp. 1-11 (2009).

5. Yang, Q.S., Qin, Q.H., Ma, L.H., Lu, X.Z., and Cui, C.Q. "A theoretical model and finite element formulation for coupled thermo-electro-chemo-mechanical media", Mechanics of Materials, 42, pp. 148-156 (2010).

6. Kuang, Z.B. "Internal energy variational principle and governing equations in electroelastic analysis", International Journal of Solids and Structures, 46, pp. 902-911 (2009).

7. Branco, J.R., Ferreira, J.A., and da Silva, P. "NonFickian delay reaction-diffusion equation: Theoretical and numerical study", Applied Numerical Mathematics, 60, pp. 531-549 (2010).

8. Peret, T., Clement, A., Freour, S., and Jacquemin, F. "Numerical transient hygro-elastic analyses of reinforced Fickian and non-Fickian polymers", Composite Structures, 116, pp. 395-403 (2014).

9. Barbeiro, S., Bardeji, S.G.H., and Ferreira, J.A. "Laplace transform-finite element method for nonFickian flows", Computer Methods in Applied Mechanics and Engineering, 261-262, pp. 16-23 (2013).

10. Kuang, Z.B. "Variational principles for generalized thermodiffusion theory in pyroelectricity", Acta Mech., 214, pp. 275-289 (2010).

11. Lord, H.W. and Shulman, Y. "A generalized dynamical theory of thermoelasticity", Journal of the Mechanics and Physics of Solids, 15, pp. 299-309 (1967).

12. Green, A.E. and Lindsay, K.A. "Thermoelasticity", Journal of Elasticity, 2, pp. 1-7 (1972).

13. Green, A.E. and Naghdi, P.M. "Thermoelasticity without energy dissipation", Journal of Elasticity, 31, pp. 189-208 (1993).

14. Guo, S.H. "The comparisons of thermo-elastic waves for Lord-Shulman mode and Green-Lindsay mode based on Guo's eigen theory", Acta Mech., 222, pp. 199-208 (2011).
15. Hosseini, S.M. and Abolbashari, M.H. "Analytical solution for thermoelastic waves propagation analysis in thick hollow cylinder based on Green-Naghdi model of coupled thermoelasticity", Journal of Thermal Stresses, 35, pp. 363-376 (2012).

16. Hosseini, S.M. "Coupled thermoelasticity and second sound in finite length functionally graded thick hollow cylinders (without energy dissipation)", Materials and Design, 30, pp. 2011-2023 (2009).

17. Hosseini, S.M. "Shock-induced thermoelastic wave propagation analysis in a thick hollow cylinder without energy dissipation using mesh-free generalized finite difference (GFD) method", Acta Mech., 224, pp. 465478 (2013).

18. Lee, Z.Y. "Generalized coupled transient thermoelastic problem of multilayered hollow cylinder with hybrid boundary conditions", International Communications in Heat and Mass Transfer, 33, pp. 518-528 (2006).

19. Xia, R., Tian, X., and Shen, Y. "Dynamic response of two-dimensional generalized thermoelastic coupling problem subjected to a moving heat source", Acta Mechanica Solida Sinica, 27, pp. 300-305 (2014).

20. Shariyat, M. "Nonlinear transient stress and wave propagation analyses of the FGM thick cylinders, employing a unified generalized thermoelasticity theory", International Journal of Mechanical Sciences, 65, pp. 24-37 (2012).

21. Shariyat, M., Lavasani, S.M.H., and Khaghani, M. "Nonlinear transient thermal stress and elastic wave propagation analyses of thick temperature-dependent FGM cylinders using a second-order point-collocation method", Applied Mathematical Modelling, 34, pp. 898-918 (2010).

22. Shariyat, M., Khaghani, M., and Lavasani, S.M.H. "Nonlinear thermoelasticity, vibration, and stress wave propagation analyses of thick FGM cylinders with temperature-dependent material properties", European Journal of Mechanics - A/Solids, 29, pp. 378-391 (2010).

23. Bagri, A. and Eslami, M.R. "A unified generalized thermoelasticity, solution for cylinders and spheres", International Journal of Mechanical Sciences, 49, pp. 1325-1335 (2007).

24. Bagri, A. and Eslami, M.R. "A unified generalized thermoelasticity formulation; application to thick functionally graded cylinders", Journal of Thermal Stresses, 30, pp. 911-930 (2007).

25. Sherief, H.H. and Abd El-Latief, A.M. "Application of fractional order theory of thermoelasticity to a $2 \mathrm{D}$ problem for a half-space", Applied Mathematics and Computation, 248, pp. 584-592 (2014).

26. Hosseini, S.A., Abolbashari, M.H., and Hosseini, S.M. "Shock induced molar concentration wave propagation and coupled non-Fick diffusion-elasticity analysis using an analytical method", Acta Mech., 225, pp. 35913599 (2014). 
27. Kumar, R. and Chawla, V. "A study of fundamental solution in orthotropic thermodiffusive elastic media", International Communications in Heat and Mass Transfer, 38, pp. 456-462 (2011).

28. Deswal, S. and Choudhary, S. "Impulsive effect on an elastic solid with generalized Thermodiffusion", Journal of Engineering Mathematics, 63, pp. 79-94 (2009).

29. Aouadi, M. "Uniqueness and reciprocity theorems in the theory of generalized thermoelastic diffusion", Journal of Thermal Stresses, 30, pp. 665-678 (2007).

30. Aouadi, M. "Qualitative aspects in the coupled theory of thermoelastic diffusion", Journal of Thermal Stresses, 31, pp. 706-727 (2008).

31. Singh, B., Singh, L., and Deswal, S. "Reflection of plane waves in thermo-diffusion elasticity without dissipation under the effect of rotation", Mechanics of Advanced Materials and Structures, 23, pp. 74-79 (2016).

32. Allam, A.A., Omar, M.A., and Ramadan, K.T. "A thermoelastic diffusion interaction in an infinitely long annular cylinder", Archive of Applied Mechanics, 84, pp. 953-965 (2014).

33. Suo, Y. and Shen, S. "Dynamical theoretical model and variational principles for coupled temperaturediffusion-mechanics", Acta Mech, 223, pp. 29-41 (2012).

34. Suo, Y. and Shen, S. "Analytical solution for onedimensional coupled non-Fick diffusion and mechanics", Archive of Applied Mechanics, 83, pp. 397-411 (2013).

35. Hosseini, S.M., Sladek, J., and Sladek, V. "Two dimensional analysis of coupled non-Fick diffusionelastodynamics problems in functionally graded materials using meshless local Petrov-Galerkin (MLPG) method", Applied Mathematics and Computation, 268, pp. 937-946 (2015).

36. Hosseini, S.M., Sladek, J., and Sladek, V. "Two dimensional transient analysis of coupled non-Fick diffusionthermoelasticity based on Green-Naghdi theory using the meshless local Petrov-Galerkin (MLPG) method", International Journal of Mechanical Sciences, 82, pp. 74-80 (2014).

37. Cohen, A.M., Numerical Methods for Laplace Transform Inversion, Springer US (2007).

\section{Biographies}

Seyed Amin Hosseini received his $\mathrm{PhD}$ degree in Mechanical Engineering from Ferdowsi University of Mashhad, Iran, in 2017. He is currently an Assistant Professor in the Mechanical Engineering Department at Chabahar Maritime University. His research interests include computational mechanics, finite element method, fracture, and thermal stresses. He has published 10 journal and conference papers.

Seyed Mahmoud Hosseini received his PhD degree in Mechanical Engineering from Amirkabir University of Technology (Tehran Polytechnic), Iran, in 2007. He started his work at Ferdowsi University of Mashhad in 2009. He is currently an Associate Professor at Ferdowsi University of Mashhad, Iran. His main focus of research is dynamic analysis and wave propagation in solids, coupled and uncoupled thermoelasticity analysis, and stochastic and reliability analysis of stress field using analytical and numerical methods (including FEM, MLPG, and GFD methods). He has more than 80 peer-reviewed journal and conference publications.

Mohammad Hossein Abolbashari is currently professor of Mechanical Engineering at Ferdowsi University of Mashhad, Iran. He received his PhD degree from the University of Saskatchewan, Canada, in 1995. He is director of the "Lean Production Engineering Research Center." He has recently joined the Industrial Engineering department of Ferdowsi University of Mashhad as an honored member. His research interests include structural optimization, computational mechanics, finite elements methods, and time-dependent materials. He has authored/coauthored more than 112 refereed journal and conference publications. He serves as the editorial board member of two refereed journals, namely, "Journal of Applied and Computational Sciences in Mechanics" and "Journal of Solid and Fluid Mechanics," which are currently published in Farsi (Persian). He is a fellow of the Iranian Society of Mechanical Engineers (ISME) and the Iranian Organization for Engineering Order of Building. 